\title{
Recovering the past history of natural recording media by Bayesian inversion
}

\author{
Tatsu Kuwatani, ${ }^{1,2,{ }^{*}}$ Hiromichi Nagao, ${ }^{3,4}$ Shin-ichi Ito, ${ }^{3}$ Atsushi Okamoto, ${ }^{5}$ Kenta Yoshida, ${ }^{1}$ and Takamoto Okudaira ${ }^{6}$ \\ ${ }^{1}$ Japan Agency for Marine-Earth Science and Technology (JAMSTEC), Yokosuka 237-0061, Japan \\ ${ }^{2}$ PRESTO, Japan Science and Technology Agency (JST), Kawaguchi, 332-0012, Japan \\ ${ }^{3}$ Earthquake Research Institute, The University of Tokyo, Tokyo 113-0032, Japan \\ ${ }^{4}$ Graduate School of Information Science and Technology, The University of Tokyo, Tokyo 113-8654, Japan \\ ${ }^{5}$ Graduate School of Environmental Studies, Tohoku University, Sendai 980-8579, Japan \\ ${ }^{6}$ Graduate School of Science, Osaka City University, Osaka 558-8585, Japan
}

(Received 4 March 2018; published 31 October 2018)

\begin{abstract}
Spatial growth patterns are natural recording media (NRMs) that preserve important historical information, which can be accessed and analyzed to reconstruct past environmental conditions and events. Here, we propose the Bayesian inversion method, which can reconstruct the evolution of target parameters from purely spatial data by extending data assimilation (DA), a method for integrating numerical simulations with time-series observations. Our method converts discrete spatial observation data to time-series data with the help of a law representing the NRM's time-evolution dynamics and Gaussian process regression, enabling us to directly compare the observations with a numerical simulation based on the DA framework. The method's effectiveness is demonstrated using a synthetic inversion problem, namely reconstructing the pressure-temperature-time $(P-T-t)$ path of a metamorphic rock from chemical composition profiles of its zoned minerals. The proposed method is broadly applicable to a wide variety of NRMs.
\end{abstract}

DOI: 10.1103/PhysRevE.98.043311

\section{INTRODUCTION}

Nature often records the dynamics of environmental parameters as structural patterns, which we call natural recording media (NRMs), just like digital pits in compact discs (CDs) and the grooves in vinyl records. Typical examples include tree rings, ice cores, lithological layers, otoliths, shells, human nails, and secular fatigue damage accumulated in materials. These structures grow over time, so each spatial position corresponds to a particular point in time. They preserve precious information, often unique direct evidence of the past physical and chemical conditions of the target system.

That said, decoding the evolution of particular parameters from NRMs is difficult, because (1) the recorded data do not directly reflect the parameters we would like to estimate, and (2) temporal information has to be simultaneously estimated from the spatial structure. It is therefore essential to develop a method of reconstructing past dynamics from the spatial structures preserved in NRMs.

Here, we propose a Bayesian method, called historytracking inversion, that extends conventional data assimilation (DA). DA is a probabilistic method that combines numerical simulations with observed data to forecast the future state

\footnotetext{
*kuwatani@jamstec.go.jp

Published by the American Physical Society under the terms of the Creative Commons Attribution 4.0 International license. Further distribution of this work must maintain attribution to the author(s) and the published article's title, journal citation, and DOI.
}

of the system [1,2]. DA was developed for meteorology and oceanology, but has increasingly been applied in many natural science fields, including seismology, biology, chemistry, and materials science [3-6]. However, the conventional DA framework can only be directly applied to situations where time-series data are available. Although there are some examples of applying DA to NRM, such as dendrochronology [7] and ice-core chronology [8], these studies transformed the NRM's spatial data into time-series data using additional existing chronological references, such as radioactive age, prior to the DA analysis. Thus, it is still difficult to apply DA techniques to general reconstruction problems involving NRM.

The proposed method develops a new type of state-space model, which lies at the heart of DA, to analyze past dynamics using only current spatial data, i.e., data from the final state of time evolution. Such datasets, where the temporal information has been lost, often occur in nature. With the help of Gaussian process regression [9], a well-known statistical interpolation technique, the state-space model can reconstruct temporal information from the spatial structure.

To illustrate how the proposed method can extract information from NRMs, we apply it to a petrological problem: estimating pressure $(P)$-temperature $(T)$-time $(t)$ paths from the composition of zones preserved in mineral grains [10]. Rocks provide us with unique direct evidence of underground geodynamic processes, such as mountain formation, volcanism, and seismicity, caused by plate subduction and collision. Petrology tries to extract various types of information from the texture of rocks, such as the size, shape, and distribution of mineral grains $[11,12]$. Even a single mineral grain in a rock 


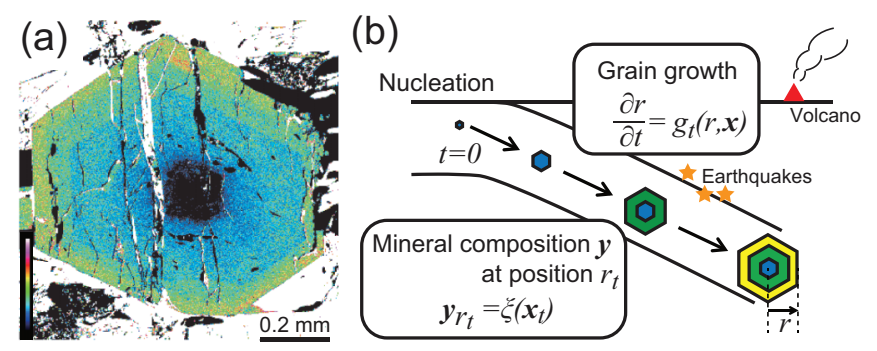

FIG. 1. (a) X-ray Mg intensity map of a garnet grain in the Sambagawa metamorphic rocks, Japan. (b) Schematic image of mineral zones. The mineral grows according to a mineral growth law, and the outer rim's mineral composition is controlled by thermodynamic variables, in this case pressure and temperature.

can record a variety of precious information, including the earliest geodynamic processes [13] and the biological activity [14] during rock formation.

Figure 1(a) shows an X-ray Mg intensity map of a garnet grain, within which we can see that the chemical composition varies as we go from the core toward the rim, reflecting the rock formation process. This spatial structure is called the compositional zoning structure. After nucleation, mineral grains grow according to a grain-growth law that depends on the governing kinetic processes and external physicochemical conditions [15] [Fig. 1(b)]. During grain growth, the chemical composition of the layer at the outer rim is also determined by the kinetic processes and external conditions present at the time.

Assuming that no processes occur within the grain to modify its composition after growth, such as diffusion or particle dissolution [16], the changes in its chemical composition in the radial direction directly record the external thermodynamic conditions at the time that layer was grown, as will be described later, including changes in temperature and pressure in many cases. As a result, it can be described as an NRM, just like vinyl records and CDs, except in this case it preserves precious underground and geodynamic history information. (In fact, a mineral's compositional zoning structure is more like a $\mathrm{CD}$, because information is recorded on CDs by starting at the center and working outwards, whereas vinyl records go in the opposite direction.) The proposed method allows us to add a time axis to the $P-T$ path by introducing an appropriate growth law for the target mineral.

In this paper, we first describe the basic framework of history-tracking inversion method. Subsequently, we apply it to the $P-T-t$ path inversion from zoned mineral. Then, we have conducted numerical experiments to evaluate the effectiveness and validity of the proposed method. We will also discuss the robustness and limitations of the proposed model, describing issues to be solved in future work so that it can be applied to a wide variety of NRMs.

\section{METHOD}

\section{A. State-space model for history-tracking inversion}

In this study, we propose a Bayesian inversion method, based on the DA framework, that can reconstruct past dynamics from the spatial structure preserved in NRMs. DA starts by (a) Data assimilation

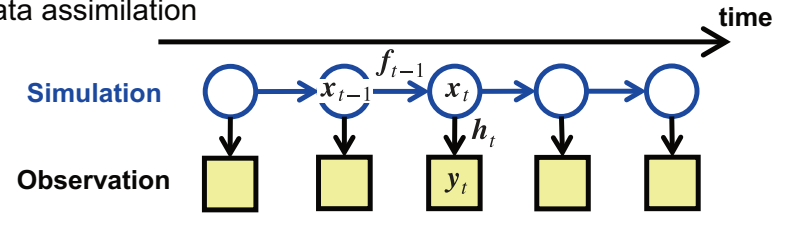

(b) History-tracking Inversion

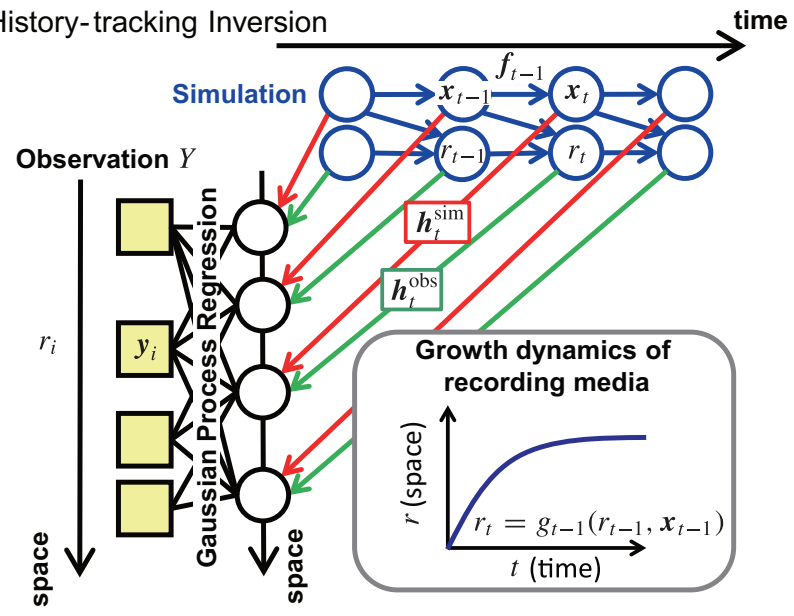

FIG. 2. Conceptual state-space models. (a) Conventional DA. (b) History-tracking inversion, as proposed in this study.

constructing a state-space model (SSM) consisting of system and observation models [Fig. 2(a)]:

$$
\begin{gathered}
x_{t}=f_{t-1}\left(x_{t-1}\right)+v_{t-1}, \\
y_{t}=h_{t}\left(x_{t}\right)+w_{t} .
\end{gathered}
$$

The system model [Eq. (1)] describes how the state $\boldsymbol{x}_{t-1}$ evolves with discretized time $t-1$ according to the given numerical model $\boldsymbol{f}_{t-1}$. The state vector $\boldsymbol{x}_{t}$ contains all the target system variables, i.e., the physical quantities at each point of the computation grid. Since $\boldsymbol{f}_{t-1}$ is not usually able to simulate all the phenomena that occur in reality, system noise $\boldsymbol{v}_{t-1}$ is introduced, following a probability density function (PDF), to absorb the impact of such imperfections in the model. In this study, according to the usual DA formalism, the system noise is assumed to be independent of the state vector. We consider that the system model is not the stochastic differential equation that includes the intrinsic probabilistic fluctuation, but one that includes the error due to model imperfectness. The formalism for stochastic differential equation will be discussed in Concluding Remarks. The observation model [Eq. (2)] compares the state $\boldsymbol{x}_{t}$ with the observed data $\boldsymbol{y}_{t}$ at time $t$, including the observation noise $\boldsymbol{w}_{t}$. The observation operator $\boldsymbol{h}_{t}$ extracts quantities from $\boldsymbol{x}_{t}$ for comparison with $\boldsymbol{y}_{t}$, such as elements of $\boldsymbol{x}_{t}$ or their transformations.

The observation model [Eq. (2)], which is usually introduced in fundamental textbooks on Bayesian time-series modeling [17], can only be used when the observed data are given as a time series. Here, however, we consider observed data taken from the spatial structure of NRMs, so we need to adapt the observation model into a more generic form. Since the observed data are given as a function of position $\boldsymbol{r}$, we 
rewrite the observation model as

$$
\boldsymbol{H}^{\mathrm{obs}}(\boldsymbol{r}, Y)-\boldsymbol{H}^{\mathrm{sim}}(\boldsymbol{r}, Z)=\boldsymbol{w}_{\boldsymbol{r}},
$$

where $\boldsymbol{H}^{\text {obs }}$ is an operator that outputs the observed data at position $\boldsymbol{r}$ from the observation dataset $Y, \boldsymbol{H}^{\text {sim }}$ is an operator that outputs the simulated values at position $\boldsymbol{r}$ from the state set $Z$, and $\boldsymbol{w}_{\boldsymbol{r}}$ represents the observation noise at position $\boldsymbol{r}$, following the PDF $\psi(\cdot)$. The observation dataset $Y$ is $\left\{\left(\boldsymbol{y}_{i}, \boldsymbol{r}_{i}^{\text {obs }}\right)\right\}_{i=1}^{N}$, where $N$ is the number of observation positions. The state set $Z$ is $\left\{\left(\boldsymbol{x}_{t}, \boldsymbol{r}_{t}^{\mathrm{sim}}\right)\right\}_{t=1}^{t_{\max }}$, where $t_{\max }$ is the last time step of the simulation.

Equation (3) can be thought of as the observation model, as it compares the simulation with the observed spatial data for the NRM. However, the equation is written for arbitrary positions $\boldsymbol{r}$, meaning that the conventional DA procedure cannot be directly applied to an SSM consisting of Eqs. (1) and (3). We therefore want to formulate the observation equation in terms of time $t$, similar to Eq. (2) for conventional DA.

In history-tracking inversion, we assume a model of how the medium has grown over time, i.e., we give an equation describing the time evolution of the data at position $\boldsymbol{r}$. Since the NRM radius evolves over time, we write the model as

$$
r_{t}=g_{t-1}\left(r_{t-1}, \boldsymbol{x}_{t-1}\right)+u_{t-1},
$$

where, as before, the stochastic variable $u_{t-1}$ represents system noise that absorbs any imperfections in the model $g_{t-1}$. Note that although the radius $r$ and time $t$ are bijective, their functional relation cannot be given analytically. One of the most important goals of this paper is to quantitatively link the position $r$ to the time $t$.

The above observation model [Eq. (3)] can be rewritten as

$$
\boldsymbol{h}_{t}^{\mathrm{obs}}\left[r_{t}, Y\right]=\boldsymbol{h}_{t}^{\mathrm{sim}}\left[\boldsymbol{x}_{t}\right]+\boldsymbol{w}_{t}\left[r_{t}, Y\right]
$$

where the operator $\boldsymbol{h}_{t}^{\text {obs }}$ extracts the observed quantity $\boldsymbol{y}_{t}$ at position $r_{t}$, as given by the simulation. Because we only have observed quantities $\boldsymbol{y}_{i}$ at sparse positions $r_{i}$, the operator calculates $\boldsymbol{y}$ values for arbitrary positions $r$ by interpolating the observation dataset $Y$. The position $\boldsymbol{r}_{t}$ is the position were the parameters observed at time $t$ are preserved in the NRM. The operator $\boldsymbol{h}_{t}^{\text {sim }}$ outputs a quantity that can be compared with $\boldsymbol{y}_{t}$ by transforming the state vector $\boldsymbol{x}_{t}$. Finally, $\boldsymbol{w}_{t}\left[r_{t}, Y\right]$ represents the observation noise at time $t$ for position $r_{t}$.

The SSM for history-tracking inversion can now be formulated using Eqs. (1), (4), and (5). Equations (1) and (4) correspond to system models describing the state vector $\boldsymbol{x}$ and reference position $r_{t}$, respectively. Equation (5) corresponds to conventional DA's observation model [Eq. (2)]. Figure 2(b) illustrates the SSM for history-tracking inversion. This observation model enables us to combine spatial observations with the temporal simulation. It simulates (i.e., tracks) the historical evolution that is recorded as observed spatial data in the NRM.

\section{B. Bayesian formulation}

The above SSM can be formulated in a Bayesian estimation framework in similar way to conventional DA, so we now present an outline of the Bayesian formulation of history-tracking inversion. Our aim is to estimate the posterior probability $p(Z \mid Y)$, namely, the conditional probability

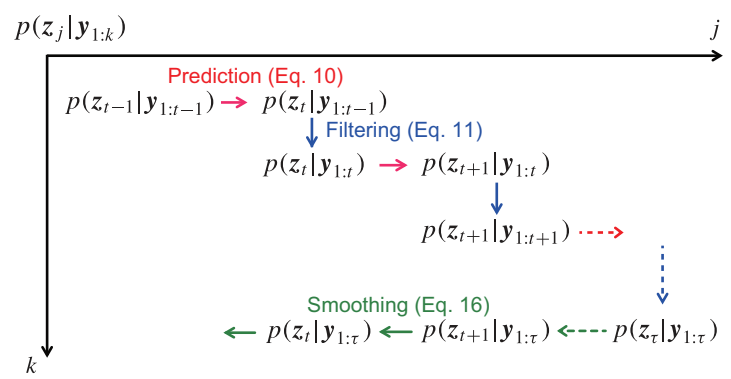

FIG. 3. Overview of predictive, filter, and smoothed PDFs in DA. Here, $p\left(\boldsymbol{z}_{j} \mid \boldsymbol{y}_{1: k}\right)$ is the conditional probability of $z$ at time $j$, given the observations $\boldsymbol{y}$ between times $1-k$.

of the state set $Z$ given the observation dataset $Y$. Bayes' theorem yields $p(Z \mid Y) \propto p(Y \mid Z) p(Z)$, where the likelihood function $p(Y \mid Z)$ measures the goodness of fit between the observation dataset and the model simulation, and the prior probability $p(Z)$ represents the prior information about the state $Z$ given by experience and knowledge. The likelihood function $p(Y \mid Z)$ is often based on the same probability density function as is followed by the observation noise $\boldsymbol{w}_{t}$ in the observation model of Eq. (5). The prior probability $p(Z)$ can be based on the initial state and the system models of Eqs. (1) and (4).

To recap, the SSM for history-tracking inversion is as follows:

$$
\begin{gathered}
\boldsymbol{x}_{t}=\boldsymbol{f}_{t-1}\left(\boldsymbol{x}_{t-1}\right)+\boldsymbol{v}_{t-1}, \\
r_{t}=g_{t-1}\left(r_{t-1}, \boldsymbol{x}_{t-1}\right)+u_{t-1}, \\
\boldsymbol{h}_{t}^{\mathrm{obs}}\left[r_{t}, Y\right]=\boldsymbol{h}_{t}^{\mathrm{sim}}\left[\boldsymbol{x}_{t}\right]+\boldsymbol{w}_{t}\left[r_{t}, Y\right],
\end{gathered}
$$

where $\boldsymbol{v}_{t}$ obeys a PDF that describes incremental changes in the target parameters $\boldsymbol{x}$, and $u_{t}$ and $\boldsymbol{w}_{t}\left[r_{t}, Y\right]$ obey PDFs that absorb any imperfections in the growth and observation models, respectively. We define the expanded system vector $z_{t}$ as

$$
\boldsymbol{z}_{t}=\left[\boldsymbol{x}_{t}, r_{t}\right]^{\top}
$$

where $T$ denotes transposition.

The goal of the inversion process is to estimate the PDFs $p\left(z_{t} \mid Y\right)$ for the observation dataset $Y=\left\{\left(\boldsymbol{y}_{i}, r_{i}\right)\right\}_{i=1}^{N}$. The operator $\boldsymbol{h}_{t}^{\text {obs }}$ extracts $\boldsymbol{y}_{t}$ from $Y$ and $r_{t}$, the radius at time $t$ as simulated by Eq. (7). Hereafter, we regard the $\boldsymbol{y}_{t}$ obtained at continuous time $t$ as the observed time-series data. Thus, we need to estimate the PDFs $p\left(z_{t} \mid \boldsymbol{y}_{1: \tau}\right)$, where $1: \tau$ is the set of discrete times 1 to $\tau$, i.e., $1: \tau=\{1,2, \ldots, \tau\}$.

These PDFs can be classified into three types depending on the relationship between the magnitudes of $t$ and $\tau$ (Fig. 3): (1) predictive PDFs when $t>\tau$; (2) filter PDFs when $t=\tau$; and (3) smoothed PDFs when $t<\tau$. In this study, smoothed PDFs represent posterior target probabilities because observations are available for later times. The one-step-ahead predictive PDF at time $t$, i.e., $p\left(z_{t} \mid \boldsymbol{y}_{1: t-1}\right)$, can be computed using the filter PDF at time $t-1$, i.e., $p\left(z_{1: t-1} \mid \boldsymbol{y}_{1: t-1}\right)$, as

$$
p\left(z_{t} \mid \boldsymbol{y}_{1: t-1}\right)=\int_{-\infty}^{\infty} p\left(z_{t} \mid z_{t-1}\right) p\left(z_{t-1} \mid \boldsymbol{y}_{1: t-1}\right) d z_{t-1} .
$$


Note that $p\left(z_{t} \mid z_{t-1}\right)$ can be calculated using the system model [Eqs. (6) and (7)]. Using the obtained predictive PDF [Eq. (10)], the filter PDF at time $t$, i.e., $p\left(z_{t} \mid \boldsymbol{y}_{1: t}\right)$, is given by

$$
p\left(z_{t} \mid \boldsymbol{y}_{1: t}\right)=\frac{p\left(\boldsymbol{y}_{t} \mid z_{t}\right) p\left(z_{t} \mid \boldsymbol{y}_{1: t-1}\right)}{\int_{-\infty}^{\infty} p\left(\boldsymbol{y}_{t} \mid z_{t}\right) p\left(z_{t} \mid \boldsymbol{y}_{1: t-1}\right) d z_{t}}
$$

where the likelihood $p\left(\boldsymbol{y}_{t} \mid z_{t}\right)$ is usually defined by using the difference between the simulated and observed values, $\Delta \boldsymbol{y}_{t}$, in place of the PDF $\psi(\cdot)$ :

$$
p\left(\boldsymbol{y}_{t} \mid z_{t}\right)=\psi\left(\Delta \boldsymbol{y}_{t}\right),
$$

where in our case $\Delta \boldsymbol{y}_{t}$ is

$$
\Delta \boldsymbol{y}_{t}=\boldsymbol{h}_{t}^{\mathrm{obs}}\left[r_{t}, Y\right]-\boldsymbol{h}_{t}^{\mathrm{sim}}\left[\boldsymbol{x}_{t}\right] .
$$

The operator $\boldsymbol{h}_{t}^{\text {obs }}$ interpolates the sparse observed composition data $Y$ to arbitrary radii $r_{t}$. To transform the scattered discrete dataset of composition observations into a continuous one, we interpolate the data using Gaussian process regression [9], which is widely used for prediction and optimization in practical fields $[18,19]$. If the normalization constant in the denominator of Eq. (11), which is equivalent to $p\left(\boldsymbol{y}_{1: t}\right)$, is difficult to compute, an unnormalized filter PDF is usually used in the numerator instead of the normalized one. Since the observation noise $\boldsymbol{w}_{t}$ is assumed to follow a Gaussian distribution, the likelihood function $p\left(\boldsymbol{y}_{t} \mid \boldsymbol{z}_{t}\right)$ is defined as

$$
p\left(\boldsymbol{y}_{t} \mid \boldsymbol{z}_{t}\right)=\frac{1}{(2 \pi)^{\frac{d}{2}}|\Sigma|^{\frac{1}{2}}} \times \exp \left[-\frac{\left(\Delta \boldsymbol{y}_{t}\right)^{\top} \Sigma^{-1} \Delta \boldsymbol{y}_{t}}{2}\right],
$$

where $d$ is the dimension of $\boldsymbol{y}_{t}$ and $\Sigma$ is the diagonal covariance matrix whose diagonal elements are the variances of the compositions, $\boldsymbol{\sigma}_{r_{t}}^{2}=\left(\sigma_{r_{t}, 1}^{2}, \sigma_{r_{t}, 2}^{2}, \ldots, \sigma_{r_{t}, d}^{2}\right)^{\top}$ :

$$
\Sigma=\operatorname{diag}\left(\sigma_{r_{t}}^{2}\right)
$$

Note that $\sigma_{r_{t}}^{2}$ is given by Gaussian process regression.

The sequential data assimilation procedure requires that the predictive and filter PDFs are computed in sequence starting from the prior PDF $p\left(z_{0}\right)$. Given the prior PDF $p\left(z_{0} \mid \boldsymbol{y}_{1: 0}\right)$ for the initial state $z_{0}$, where $\boldsymbol{y}_{1: 0}$ is an empty set, Eqs. (10) and (11) can be used to calculate the predictive and filter PDFs at all points in time. The smoothed PDFs can be computed by repeatedly applying the following equation to the obtained predictive and filter PDFs from $t=\tau-1$ to $t=1$ :

$$
p\left(z_{t} \mid \boldsymbol{y}_{1: \tau}\right)=p\left(z_{t} \mid \boldsymbol{y}_{1: t}\right) \int_{-\infty}^{\infty} \frac{p\left(z_{t+1} \mid z_{t}\right) p\left(z_{t+1} \mid \boldsymbol{y}_{1: \tau}\right)}{p\left(z_{t+1} \mid \boldsymbol{y}_{1: \tau}\right)} d z_{t+1} \text {. }
$$

\section{APPLICATION TO $P-T-t$ PATH INVERSION FROM ZONED MINERAL}

Petrologists decode the history of the rock-forming process from the compositional zoning structures of minerals. For example, metamorphic petrologists have estimated the changes in pressure and temperature (the $P-T$ path) experienced by metamorphic rocks $[10,20,21]$. The $P-T$ path can be used to infer movements of mass and heat at depths of up to a couple of hundred kilometers, leading to the construction of tectonic models of plate subduction and collision. Likewise, igneous petrologists have estimated the processes and conditions (pressure, temperature, and volatile content) present in the magma chambers of volcanoes [22-25]. This can be used to infer information about movements, compositional evolution, and recharge events in magma chambers, and may contain clues to the mechanisms that trigger eruptions.

In general, previous studies have either ignored time evolution [26] or used an independent time scale, such as that given by radiogenic isotopes [27]. Although some studies have tried to constrain the time evolution, they have analyzed the grain growth and mineral composition separately, to constrain the time evolution and the external physicochemical conditions, respectively [28,29].

In contrast, we consider the problem of estimating the pressure-temperature-time $(P-T-t)$ history of rock formation from a one-dimensional profile of the chemical composition of garnet grains. In the $P-T$ inversion problem, the target variables that evolve with discretized time $t$ are the grain radius $r$, temperature $T$ and pressure $P$. For the forward model, we assume a grain-growth law (governing increases in radius) and thermodynamic equations (relating the chemical composition to the temperature and pressure at the time).

\section{A. Simulation model}

Unlike most normal DA problems, we do not assume an explicit dynamical model for the time evolution of pressure and temperature in this study, i.e. the function $\boldsymbol{f}_{t}$ in the system model [Eq. (1)]. Instead, our method adopts a randomwalk model for time evolution, with the temperature $T_{t}$ and pressure $P_{t}$ changing probabilistically according to a Gaussian distribution. Their system models thus become

$$
\begin{array}{ll}
T_{t}=T_{t-1}+\Delta T_{t-1}, & \Delta T_{t-1} \sim N\left(0, \sigma_{\Delta T}^{2}\right), \\
P_{t}=P_{t-1}+\Delta P_{t-1}, & \Delta P_{t-1} \sim N\left(0, \sigma_{\Delta P}^{2}\right),
\end{array}
$$

where $\Delta T_{t-1}$ and $\Delta P_{t-1}$ are the probabilistic temperature $T_{t-1}$ and pressure $P_{t-1}$ changes, respectively, both of which follow a Gaussian distribution. The equations use expediently probabilistic formalism using additive probabilistic parameters to represent the continuous changes in temperature and pressure. It is noted that the driving force of temperature and pressure changes is not the intrinsic probabilistic fluctuation but the geodynamic tectonic movements. To allow large jumps in pressure and temperature over the course of the $P-T-t$ path, the random walk's variance is set to be larger than would be expected for the normal time evolution of pressure and temperature.

Since the grain is assumed to grow concentrically with time, the grain radius $r$ corresponds to the spatial position $\boldsymbol{r}$. The diffusion-controlled grain-growth law used is [15]

$$
\frac{\partial r}{\partial t}=k \frac{1}{r} \exp \left(-\frac{E_{\mathrm{a}}}{R T}\right)
$$

where $k$ is the rate constant and $E_{\mathrm{a}}$ is the activation energy. We discretize Eq. (18) with respect to time as

$$
\begin{aligned}
r_{t}^{2} & =r_{t-1}^{2}+2 k \exp \left(-\frac{E_{a}}{R T_{t-1}}\right)+\Delta r_{t-1}^{2}, \\
\Delta r_{t-1}^{2} & \sim N\left(0, \sigma_{\Delta r^{2}}^{2}\right) .
\end{aligned}
$$


This time-evolution equation can be used to model the reference position for the NRM [Eq. (4)] in our proposal.

Based on these, we can define the state vector and system noise as

$$
\begin{gathered}
\boldsymbol{z}_{t}=\left[T_{t}, P_{t}, r_{t}^{2}\right]^{\top}, \\
\boldsymbol{\varepsilon}_{t}=\left[\Delta T_{t}, \Delta P_{t}, \Delta r_{t}^{2}\right]^{\top} .
\end{gathered}
$$

\section{B. Observation model}

We also assume that the chemical composition of the growing garnet grain's outer rim is in equilibrium with those of all the other minerals at the time. In the present study, we assume the thermodynamic system of Kuwatani et al. [26]. This determines the chemical compositions of the constituent minerals, given the temperature $T$ and pressure $P$, based on chemical stoichiometric constraints and the following mass action law:

$$
0=\Delta \mu^{\circ}(P, T)+R T \ln K_{\mathrm{eq}}\left(X_{1}, X_{2}, \ldots\right),
$$

where $\Delta \mu^{\circ}$ is the standard free energy change for a reaction, $K_{\text {eq }}$ is the equilibrium constant, and $X_{1}, X_{2}, \ldots$ are the molar compositions.

For the NRM, we use a garnet grain consisting of the solid solution ( $\mathrm{Fe}, \mathrm{Ca}, \mathrm{Mg})_{3} \mathrm{Al}_{2} \mathrm{Si}_{3} \mathrm{O}_{12}$. The thermodynamic forward model for this can be derived as

$$
\begin{aligned}
& X_{\mathrm{Fe}}=\xi_{\mathrm{Fe}}(T, P), \\
& X_{\mathrm{Ca}}=\xi_{\mathrm{Ca}}(T, P),
\end{aligned}
$$

where $\mathrm{Fe}$ and $\mathrm{Ca}$ represent the almandine $\left(\mathrm{Fe}_{3} \mathrm{Al}_{2} \mathrm{Si}_{3} \mathrm{O}_{12}\right)$ and grossular $\left(\mathrm{Ca}_{3} \mathrm{Al}_{2} \mathrm{Si}_{3} \mathrm{O}_{12}\right)$ components of the garnet solid solution, respectively. Broadly, the changes in $X_{\mathrm{Fe}}$ and $X_{\mathrm{Ca}}$ are sensitive to the changes in $T$ and $P$, respectively. We use the above equations directly for the simulation operator $\boldsymbol{h}_{t}^{\text {sim }}$ that outputs the observation parameters $\boldsymbol{y}_{t}^{\text {sim }}$ based on the system parameters $\boldsymbol{x}_{t}$ in the observation model [Eq. (5)].

Using the latter two forward models, the composition at radius $r(t)$ can be calculated for arbitrary $P-T-t$ trajectories. In practice, however, observed mineral compositions are sparse and suffer from measurement noise due to the various analytical and sample conditions described in next section.

The observed data are the mineral molar compositions $Y=\left\{\left(X_{\mathrm{Fe}, i}, X_{\mathrm{Ca}, i}, r_{i}\right)\right\}_{i=1}^{N}$. The observation model Eq. (8) links the observed spatial data $Y$ with the simulated temporal data $z_{t}$, allowing the likelihood function to be formulated. The observation operator $\boldsymbol{h}_{t}^{\text {obs }}$ extracts the compositional data $X_{\mathrm{Fe}, t}^{\mathrm{obs}}, X_{\mathrm{Ca}, t}^{\mathrm{obs}}$ at $r=r_{t}$ from the sparse observation data, interpolated using Gaussian process regression. The simulation operator $\boldsymbol{h}_{t}^{\text {sim }}$ calculates the simulated composition data $X_{\mathrm{Fe}, t}^{\mathrm{sim}}, X_{\mathrm{Ca}, t}^{\mathrm{sim}}$ using the thermodynamic functions [Eqs. (23a) and (23b)] and the simulated $T_{t}$ and $P_{t}$ values. The observation operator $\boldsymbol{w}_{t}^{\text {obs }}$ calculates the variances of the compositional data $X_{\mathrm{Fe}, t}^{\mathrm{obs}}, X_{\mathrm{Ca}, t}^{\mathrm{obs}}$ at $r=r_{t}$ using Gaussian process regression.

\section{NUMERICAL EXPERIMENTS}

\section{A. Problem setting}

To evaluate the effectiveness and validity of the proposed method, we applied it to several synthetic datasets. The details of the problem setting are described in the Appendix. The synthetic garnet grain grew according to the assumed diffusion-controlled grain-growth law [Eq. (18)] along the assumed $P-T-t$ trajectory, as shown in Figs. 4(a)-4(c). This trajectory was synthesized to represent the rock going underground along with a subducting oceanic crust. The garnet grain grew to a size of about $7.2 \mathrm{~mm}$ during 2.0 million years (Myr). The chemical compositions, $X_{\mathrm{Fe}}\left(r_{t}\right)$ and $X_{\mathrm{Ca}}\left(r_{t}\right)$, were obtained for each radius $r_{t}$ at each time $t$ during this growth period using the thermodynamic forward model [Eqs. (23a) and (23b)].

Due to the analytical and sample conditions, the composition measurements were sparse. Figures 4(e) and 4(f) show the observed composition profiles for the almandine $\left(X_{\mathrm{Fe}}\right)$ and grossular $\left(X_{\mathrm{Ca}}\right)$ components, respectively. These show that the almandine component decreased approximately monotonously from core to rim, whereas the grossular component initially decreased before converging to a constant value. In addition, Gaussian noise, with a mean of 0 and a standard deviation of 0.005 , was added to both observed components. The assumed noise magnitudes $(0.005)$ broadly correspond to those of the analytical uncertainties, which have been largely derived from error statistics for simple electron microprobe counting [30,31].

Finally, we implemented Bayesian history-tracking inversion using a sequential Monte Carlo method, also known as a particle filter [17,32] (see the Appendix). For this method, 4000 particles were used and the smoothing lag was 10 steps. The mean values of the smoothed distribution were used for the estimated values.

\section{B. Estimation of $\boldsymbol{P}-\boldsymbol{T}-\boldsymbol{t}$ path}

The estimated results are shown in Figs. 5 and 6, as well as in the Supplemental Material [33]. To compare the observations with the simulated temporal evolution of the state-space model [Eq. (5)], observation data were needed for arbitrary radii at each simulated time step. The discrete observation data $\left\{\boldsymbol{X}_{i}^{\text {obs }}, r_{i}\right\}$ were therefore spatially interpolated using Gaussian process regression (Fig. 5). The Gaussian kernel function, $\operatorname{kernel}\left(r, r^{\prime}\right)=\sigma_{f}^{2} \exp \left[-\left(r-r^{\prime}\right)^{2} / \sigma_{l}^{2}\right]$, was adopted because it is a basic and tractable function. In addition, the kernel function is physically consistent with the Gaussian random walk of the target parameters. The hyperparameter estimation using the framework of maximization of marginal likelihood gave $\left(\sigma_{f}, \sigma_{l}\right)=(0.1168,0.0044)$ for $X_{\mathrm{Fe}}$ and $\left(\sigma_{f}, \sigma_{l}\right)=$ $(0.0257,0.0037)$ for $X_{\mathrm{Ca}}$, respectively. Figure 5 showed that the mean values, corresponding to $\boldsymbol{h}_{t}^{\text {obs }}$, passed close to all the observed values. In Gaussian process regression, the standard deviation $\sigma_{r_{t}}$ can vary along the profile. When the observation points were more widely spaced, the $\pm 2 \sigma$ range was larger, and vice versa. This is consistent with our natural intuition that the uncertainty in the chemical composition will vary depending on the observation point density. The standard deviations were $0.015-0.02$ for $X_{\mathrm{Fe}}$ and $0.01-0.015$ for $X_{\mathrm{Ca}}$. The 
(a)

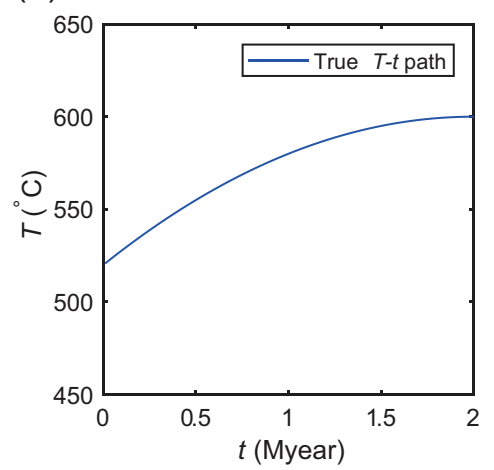

(d)

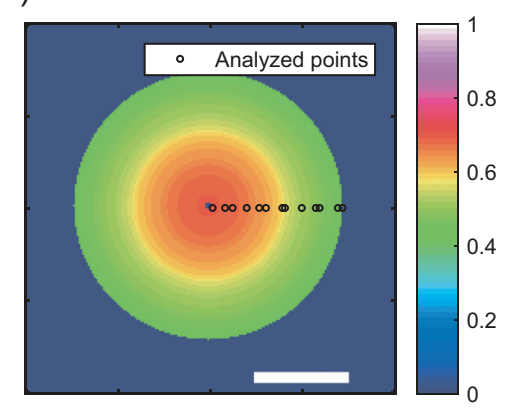

(b)

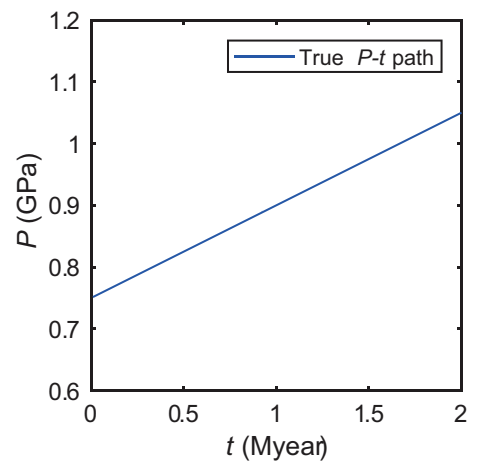

(e)

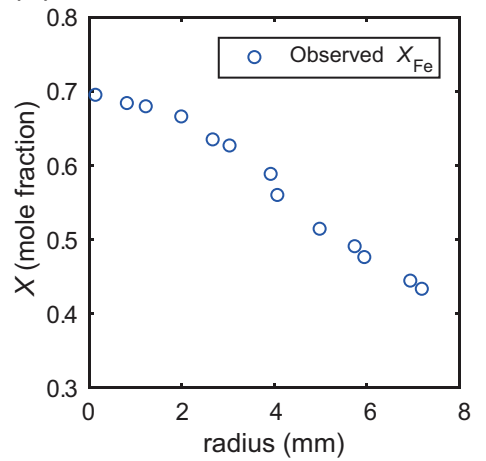

(c)

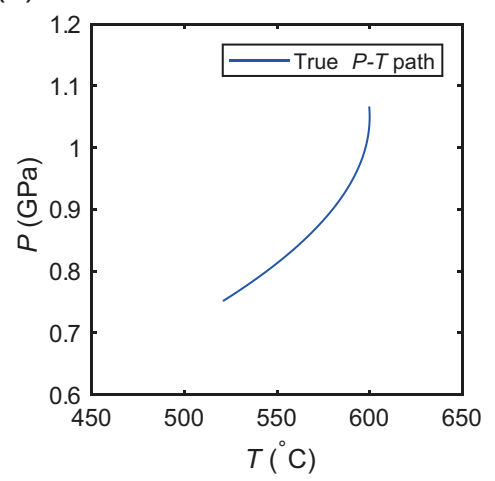

(f)

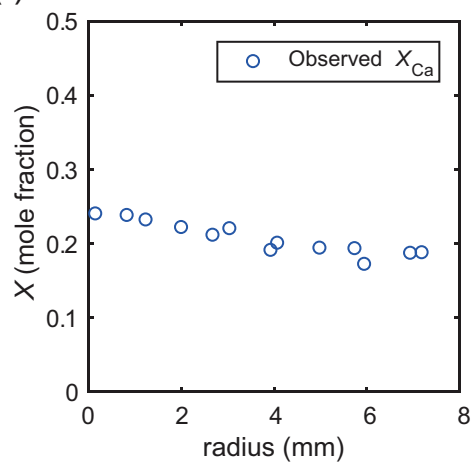

FIG. 4. Problem setup for the numerical experiments. (a) True $T-t$ path. (b) True $P-t$ path. (c) True $P-T$ path. (d) Composition of $X_{\mathrm{Fe}}$ in a synthetic garnet grain. The scale bar represents $5.0 \mathrm{~mm}$. (e) Observed composition profile for $X_{\mathrm{Fe}}$. (f) Observed composition profile for $X_{\mathrm{Ca}}$.

likelihood function for the observation model [Eq. (5)] was then calculated using these mean and standard deviation values.

Figures 6(a), 6(b) and 6(d) show the estimated time evolution of the temperature, pressure, and grain size, respectively. The regions within the upper and lower $2 \sigma$ limits calculated using the Monte Carlo particles are also shown. The temperature and grain size estimates are good fits to the true profiles. However, the pressure estimates deviate slightly from the true profile, although they roughly reproduce its shape. The differences between the true and estimated values were evaluated using the root mean squared error (RMSE), defined as

$$
\begin{aligned}
\operatorname{RMSE}_{T} & =\sqrt{\frac{\sum_{t=1}^{t_{\max }}\left(T_{t}^{\text {est }}-T_{t}^{\text {true }}\right)^{2}}{t_{\text {max }}},}, \\
\operatorname{RMSE}_{P} & =\sqrt{\frac{\sum_{t=1}^{t_{\max }}\left(P_{t}^{\text {est }}-P_{t}^{\text {true }}\right)^{2}}{t_{\text {max }}}} .
\end{aligned}
$$

The errors were $2.5^{\circ} \mathrm{C}, 0.019 \mathrm{GPa}$, and $0.12 \mathrm{~mm}$ for the temperature, pressure, and radius of the growing mineral, respectively. The ranges between the upper and lower limits were much smaller than the overall changes for the temperature and grain size, but significantly larger for the pressure. This is due to the fact that the observed composition is much less sensitive to pressure than to temperature [26].

Figure 6(c) shows the estimated $P-T$ path, indicating that its convex shape was broadly reproduced. Figures 6(e) and 6(f) show the Monte Carlo particle distributions for the obtained smoothed distribution, $h_{t}^{\mathrm{sim}}\left(\boldsymbol{x}_{t}\right)$. These show that the filter particles were also concentrated near the observed values, within the $\pm 2 \sigma$ range calculated by the Gaussian process. From the above, we can conclude that, at least for this problem, the numerically calculated state-space model succeeded in reproducing the $P-T-t$ path based on the garnet grains observed composition profile.

One potential issue is that the estimated path may depend on how the random observation noise values are calculated. To investigate how the estimates depend on the way the random noise is generated, we estimated paths for 20 different random number generation methods. The results show that, while the shapes of the paths are different, they are concentrated near the true $P-T$ path, suggesting that the estimates are robust against the assumed level of measurement noise.
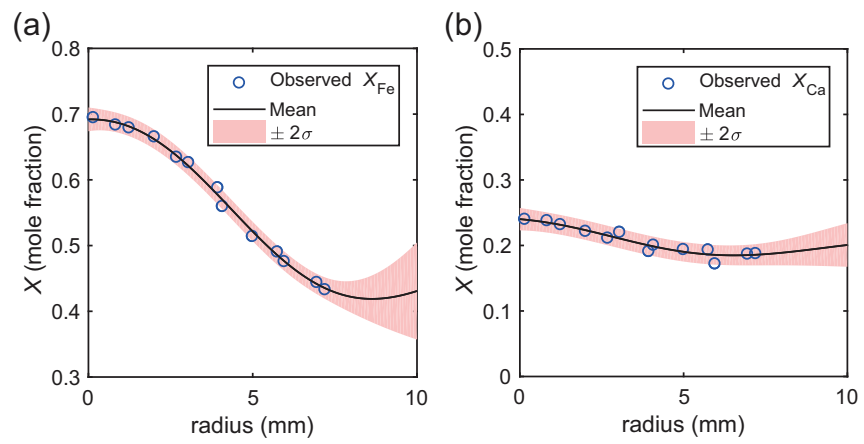

FIG. 5. Results of interpolation using Gaussian process regression for the observed compositions, $X_{\mathrm{Fe}}$ and $X_{\mathrm{Ca}}$, showing the means and $\pm 2 \sigma$ limits. 
(a)

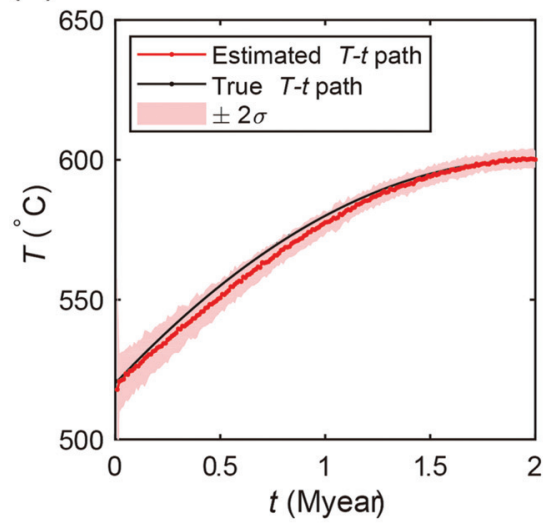

(d)

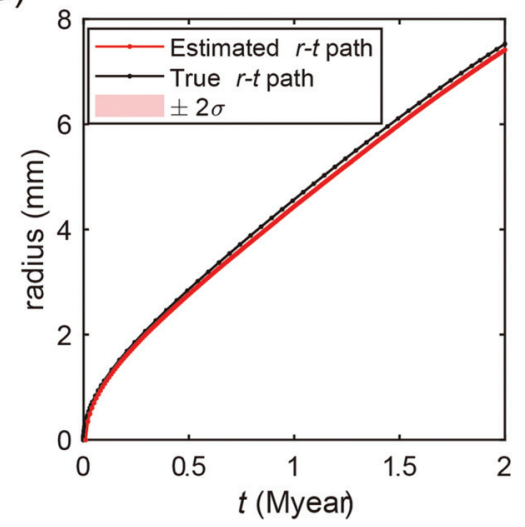

(b)

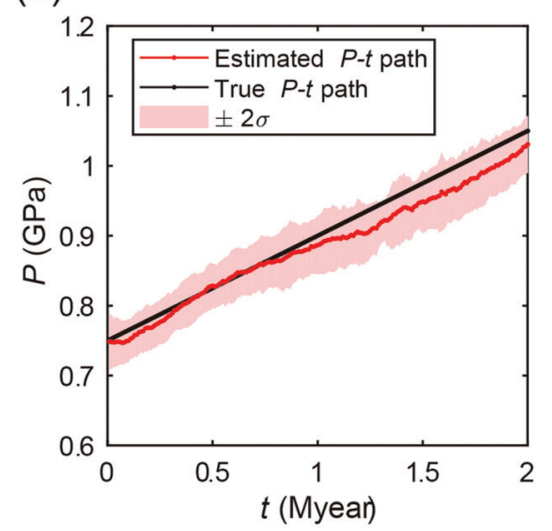

(e)

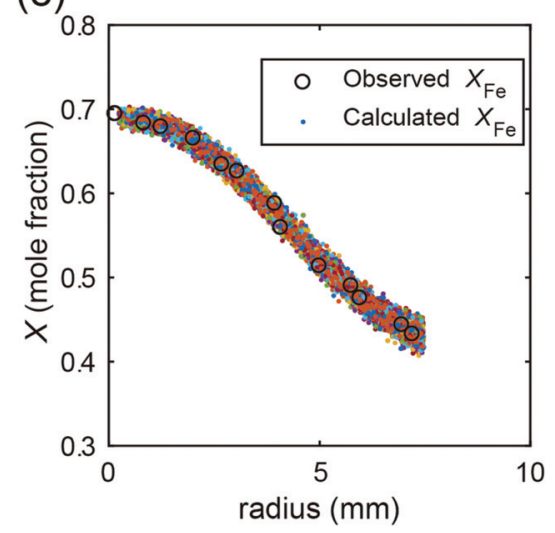

(c)

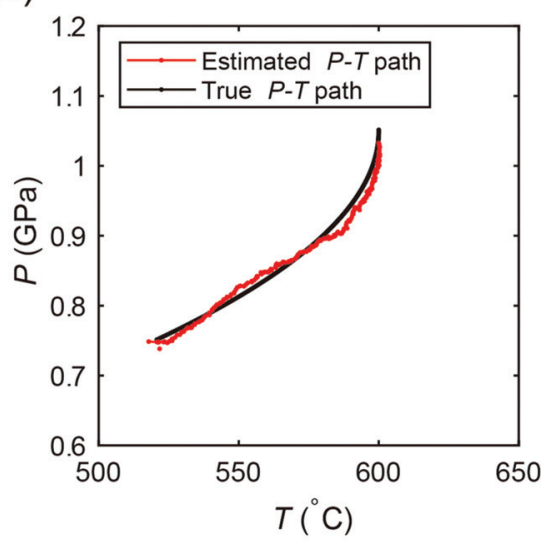

(f)

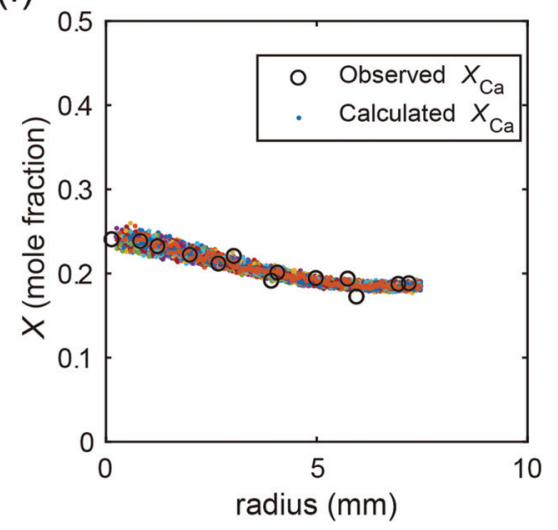

FIG. 6. Results for the numerical experiments. (a) Estimated $T-t$ path. (b) Estimated $P-t$ path. (c) Estimated $P-T$ path. (d) Radius of the growing grain. (e) Composition profile for $X_{\mathrm{Fe}}$. (f) Composition profile for $X_{\mathrm{Ca}}$. In (a)-(d), the estimated and true paths shown as red and black lines, respectively. In (a), (b), and (d), the estimated $\pm 2 \sigma$ uncertainties are also shown. In (e) and (f), the small colored points correspond to the compositions calculated using Monte Carlo particles for the smoothed PDFs, while the black circles are the observed compositions. To show the overall distributions, only 100 points are shown, randomly selected from the 4000 Monte-Carlo particles used.

\section{Sensitivity of the estimates to noise magnitude and measurement sparsity}

In practice, the magnitude of the measurement noise and/or other uncertainties and the number of measurement points can vary widely depending on the problem and sample conditions. In this section, we investigate how these affect estimation accuracy. The true $P-T-t$ path was the same as before. The noise magnitude (standard deviation of the mole fractions) $\sigma_{X}$ was $0.0,0.0025,0.005,0.0075,0.010,0.0125$, or 0.015 , and the noise magnitudes of $X_{\mathrm{Fe}}$ and $X_{\mathrm{Ca}}$ were assumed to be equal. The number of measurement points $N_{\text {obs }}$ was 5,10 , $15,20,30,40$, or 50 . To decrease the effect of uncertainty due to how the measurement points were scattered across the profile, the interval between measurement points was kept constant. Again, the difference between the true and estimated values was evaluated using the RMSE. For each combination of $\sigma_{X}$ and $N_{\text {obs }}$ values, mean $\mathrm{RMSE}_{T}$ and $\mathrm{RMSE}_{P}$ values were calculated over 100 trials.

Figure 7 shows the relationships between the RMSEs and the observation noise $\sigma_{X}$. Both $\mathrm{RMSE}_{T}$ and $\mathrm{RMSE}_{P}$ increased with the magnitude of the noise $\sigma_{X}$ for each number of observations. When there was no noise, the RMSEs were small, about $1{ }^{\circ} \mathrm{C}$ for temperature and $0.004 \mathrm{GPa}$ for pressure, respectively. They remained similar to each other even for different numbers of observations, indicating that such small errors are derived not from observation noise but from the (a)

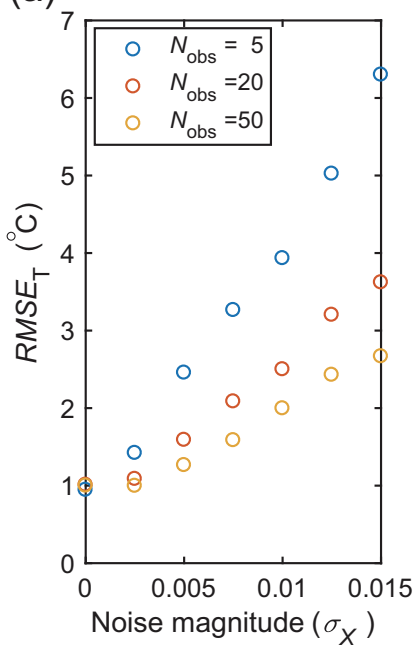

(b)

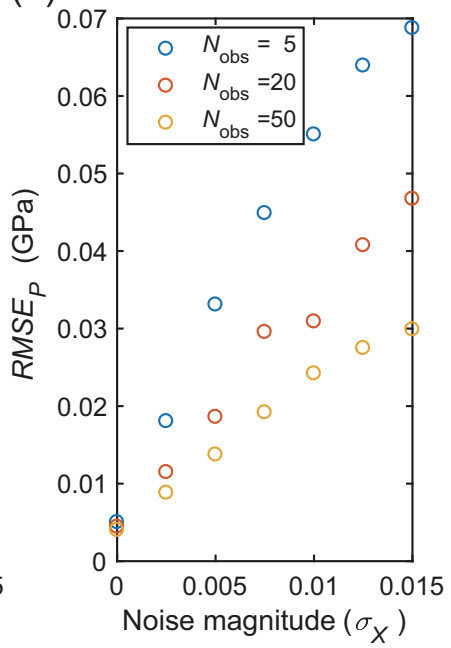

FIG. 7. Relationship between RMSE and measurement noise. (a) $T-t$ path. (b) $P-t$ path. The blue, red, and orange circles represent 5 , 20 , and 50 measurement points, respectively. 
(a)

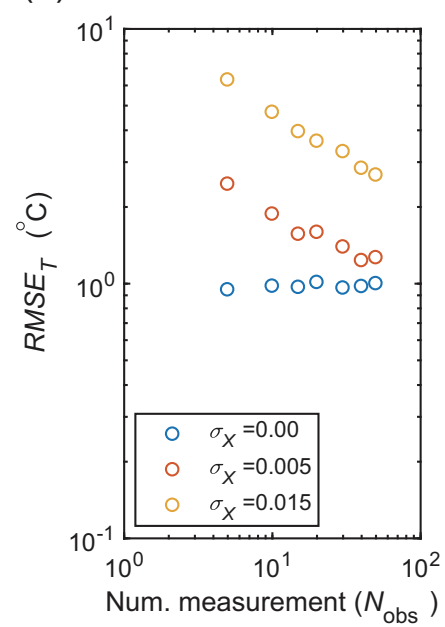

(b)

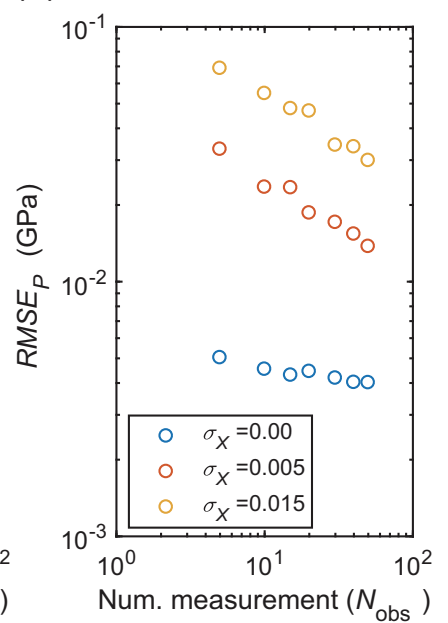

FIG. 8. Relationship between RMSE and the number of measurement points. (a) $T-t$ path. (b) $P-t$ path. The blue, red, and orange circles represent noise standard deviations of $0.000,0.005$, and 0.015 , respectively.

intrinsic ill-posedness of the problem, which is independent of the observations. When the noise was greater than 0.0025 , the estimation error increased approximately linearly with the noise magnitude, and the slopes of the fitted lines became steeper as the number of observations decreased. These results indicate that the proposed method is capable of estimating the true parameters for the given noise levels and numbers of observations.

The highest noise level (0.015) corresponds to the cumulative effect of several uncertainties, such as tiny inclusions causing composition fluctuations and analytical uncertainties [31,34]. Here, the RMSEs were large, with temperature errors of $6.3^{\circ} \mathrm{C}$ for $N_{\text {obs }}=5$ and $2.7^{\circ} \mathrm{C}$ for $N_{\text {obs }}=50$, and pressure errors of $0.07 \mathrm{GPa}$ for $N_{\mathrm{obs}}=5$ and $0.003 \mathrm{GPa}$ for $N_{\mathrm{obs}}=50$.

Figure 8 shows logarithmic plots of the relationship between the RMSEs and the number of observations $N_{\text {obs }}$. For both pressure and temperature, the RMSEs were proportional to $\sqrt{N}$, except when there was no noise, indicating that the system followed the law of large numbers with respect to the number of observations. When there was no noise, the RMSEs did not depend on the number of measurements for temperature and only very weakly for pressure, suggesting the presence of estimation errors that were not due to observation noise.

In general, for the whole region, except around noise magnitudes $>0.01$ and $<10$ measurement points, convex downward $P-T$ paths could be broadly reconstructed. This suggests that the estimates were very accurate from the viewpoint of metamorphic petrology. Taken together, these results show that our proposed method is effective against both sparseness and noise of observation.

\section{CONCLUDING REMARKS}

We have proposed a method for reconstructing the time evolution of unknown parameters from spatial observation data based on a data assimilation framework. In conventional DA, a state-space model is constructed to relate a numerical

simulation to temporal observations. When we only have spatial observations that have been recorded on natural recording media (NRMs), we have to translate this spatial data to temporal information. The proposed method provides a continuous change of variables by interpolating between discrete spatial observations, enabling us to compare the observations directly with the state-space model's numerical simulation. This interpolation is achieved using Gaussian process regression, and the obtained probabilistic function can be used directly as the likelihood function for the observation process in the state-space model.

The proposed method was applied to a synthetic inversion problem, namely reconstructing the $P-T-t$ path of a metamorphic rock from the chemical composition profile of its zoned minerals. In this test, the true $P-T-t$ path was successfully reconstructed from noisy observational data. With our method, the estimation accuracy can also be evaluated for each unknown variable, which is important for scientific work. We evaluated the effectiveness of the model for a variety of measurement noise levels and numbers of measurements, finding that the proposed method was suitable for application to real petrology problems using natural samples.

The proposed method can track the history of a system's evolution based on an NRM, and has a wide range of applications, including spatial growth patterns (such as tree rings) and geological layers. In particular, it could be useful in cases where there is limited temporal information, in terms of either relative or absolute age, because it is based on a probabilistic approach. In the earth sciences, a common and important problem is reconstructing the evolution of target parameters from only a few signatures that can be used to determine the absolute age. One such example is petrological inversion problems involving mineral grains that host several inclusions, such as zircon and monazite, that can be used for Th-U-Pb radiometric dating. Another is the environmental problem of identifying the deposits from events such as tsunamis and storm surges from borehole cores that include volcanic tephra key layers and radiocarbon-datable organic matter.

Although the proposed model could be applied to various NRMs, the limits of its applicability must be carefully noted. In particular, one fundamental requirement is that the spatial position $r$ corresponds bijectively to the time $t$. Even if this was once true, it may not be true any more, as the NRM has become degraded and broken. For example, the composition profiles of the zoned minerals in high-temperature rocks can become blurred by intra-crystal diffusion of elements. When such a process changes the original zoning profile, it makes the problem very difficult. In addition, in this study we started by assuming the forward model and the parameters for the NRM's growth law [Eq. (4)], but in natural science problems these are often unknown and must be determined.

The proposed method must therefore be developed so that it can flexibly adapt to each individual problem. If it is to be applied to problems that include multiple complex processes, processes for selecting appropriate models and estimating the hyperparameters will be required $[35,36]$. For precise analysis, it will also be important to broaden the range of observation data that can be considered. For example, we have only considered a 1D composition profile of a single grain in this study. In future work, 2D composition data for many 
grains will be utilized to precisely reconstruct the history of rock formation.

For theoretical aspects, there still are open challenges to improve the method for future studies. In this study, in accordance with the usual DA formalism, we assume the additive noise, which is independent of state variables in system model, to calculate the time evolution of parameters. In this case, discrete difference equations of a system model, such as Eqs. (1) and (4), always approach the true continuous formulation asymptotically. On the other hand, in cases that the system model is written by stochastic differential equations that include intrinsic probabilistic fluctuation, the noises are often multiplicative and dependent on the state variables.

In such cases, discrete difference equations should be carefully formulated for the stochastic integration because the discrete time formulation has no trivial continuum limit. There are two representative stochastic integral formulations: One is Ito-type formalism that evaluates the integral based on the present information and the other is Stratonovich-type formalism that evaluates the integral based on the present and future at one-step ahead of time information [37]. The appropriate formalism which best fits the target and problem should be chosen.

In addition, there would be a room for improving the method dealing with the discrete observation data in NRMs. In this study, the Gaussian process regression is adopted for interpolating the discrete observation data. However, ideally, it is hoped that the likelihood function would be calculated without ad hoc assumption for selecting an interpolation method in the Bayesian framework. It is important to improve the method in terms of both aspects of physical modeling and mathematical algorithm.

The proposed approach can serve as a basic inversion framework for reconstructing dynamics from NRMs. Inversion problems where useful information must be extracted from NRMs crop up in a wide variety of natural science fields. Since the problem considered here is a very simple case, we will have to develop and adjust the proposed model so that it can be applied to the complex problems that occur in practice. The fact that it can be applied to such a wide variety of problems highlights the power and universality of the Bayesian data-driven approach, which could become a key technology for the natural sciences [38].

\section{ACKNOWLEDGMENTS}

We thank W. Nishikanbara for kindly providing x-ray maps of a garnet grain. This work was partly supported by JST PRESTO (Grant No. JPMJPR1676) and JST CREST (Grant No. JPMJCR1761), the Initiative for High-Dimensional DataDriven Science through Deepening of Sparse Modeling by MEXT KAKENHI (Grants No. JP25120005 and No. JP16H01536), the Cooperative Research Program of the Earthquake Research Institute, University of Tokyo (Grant No. ERI JURP 2018-B-01), and JSPS KAKENHI (Grants No. JP15K20864 and No. JP17H01703).

\section{APPENDIX A: SEQUENTIAL MONTE CARLO METHOD}

Several algorithms are available for computing Eqs. (10)(16) numerically. The most classical one is the Kalman filter (KF), which can be used when both the model $f_{t}$ and observation operator $\boldsymbol{h}_{t}$ are given as matrices, and all PDFs appearing in Eqs. (10)-(16) are normal distributions. Equations (10)-(16) can all be reduced to linear algebraic equations under such an assumption, so the KF just has to compute the mean vector and covariance matrix for each of the normal distribution functions. However, such a strict and limiting assumption is inappropriate in most practical cases.

In contrast, particle filters (PFs), which are based on Monte Carlo computation, are applicable to a wide variety of situations. In the PF approach, the PDF $p(z)$ is approximated by an ensemble of realizations:

$$
p(z) \simeq \frac{1}{N} \sum_{i=1}^{N} \delta\left(z-z^{(i)}\right),
$$

where $z^{(i)}(i=1, \ldots, N)$ are the realizations, called "particles," and $\delta(\cdot)$ denotes the Dirac $\delta$ function. In this study, each particle represents a candidate solution set consisting of pressure, temperature, and radius values. This ensemble approximation reduces the prediction formula [Eq. (10)] to

$$
\boldsymbol{z}_{t \mid t-1}^{(i)}=f_{t}\left(z_{t-1 \mid t-1}^{(i)}\right)+\boldsymbol{v}_{t}^{(i)} \text { for } i=1, \ldots, N,
$$

where $z_{t \mid t-1}^{(i)}$ and $z_{t-1 \mid t-1}^{(i)}(i=1, \ldots, N)$ denote the ensembles approximating the predictive and filter PDFs, i.e.,

$$
\begin{aligned}
p\left(z_{t} \mid \boldsymbol{y}_{1: t-1}\right) & \simeq \frac{1}{N} \sum_{i=1}^{N} \delta\left(z-z_{t \mid t-1}^{(i)}\right), \\
p\left(z_{t-1} \mid \boldsymbol{y}_{1: t-1}\right) & \simeq \frac{1}{N} \sum_{i=1}^{N} \delta\left(z-z_{t-1 \mid t-1}^{(i)}\right) .
\end{aligned}
$$

As Eq. (A2) shows, the ensemble for the predictive PDF is obtained by simply applying the given numerical model to each particle of the filter PDF ensemble. This means that parallel computation can be used to accelerate the calculation, even when the computational cost for the given model $\boldsymbol{f}_{t}$ is large. Substituting Eq. (A3) into Eq. (11), the filter PDF at time $t$ can be written as

$$
p\left(z_{t} \mid \boldsymbol{y}_{1: t}\right) \simeq \sum_{i=1}^{N} \rho_{t}^{(i)} \delta\left(z_{t}-z_{t \mid t-1}^{(i)}\right),
$$

where $\rho_{t}^{(i)}$ is the normalized likelihood of the observation data $\boldsymbol{y}_{t}$, i.e.,

$$
\rho_{t}^{(i)}=\frac{p\left(y_{t} \mid z_{t \mid t-1}^{(i)}\right)}{\sum_{j=1}^{N} p\left(\boldsymbol{y}_{t} \mid z_{t \mid t-1}^{(j)}\right)} .
$$

Equation (A5) shows that the filter PDF ensemble is obtained by resampling the particles of the predictive PDF ensemble with replacement, with probabilities given by the likelihood [Eq. (A6)]. Several algorithms have been proposed to effectively compute Eq. (A5) with the given weights [Eq. (A6)]. In this study, we have adopted the residual systematic resampling method, which has a computational cost of $O(N)$.

A smoothing algorithm for estimating the smoothed PDF $p\left(z_{t} \mid y_{t+L}\right)$, where $L$ is called the "lag", was introduced by Kitagawa (2010). The key point is to calculate how many particles of the filtered PDF $p\left(z_{t} \mid y_{t}\right)$ survive at time $t+L$. 
The smoothed PDF ensemble at time $t$,

$$
p\left(z_{t} \mid \boldsymbol{y}_{1: t+L}\right) \simeq \frac{1}{N} \sum_{i=1}^{N} \delta\left(z-z_{t \mid t+L}^{(i)}\right),
$$

can be obtained by resampling the filtered PDF ensemble at time $t$, weighted by the number of particles surviving at $t+L$, namely,

$$
p\left(z_{t} \mid \boldsymbol{y}_{1: t+L}\right) \simeq \frac{1}{N} \sum_{i=1}^{N} \lambda_{t+L}^{(i)} \delta\left(z_{t}-z_{t \mid t}^{(i)}\right),
$$

where $\lambda_{t+L}^{(i)}$ is the normalized number of surviving particles at time $t+L$ that were generated from the $i$ th particle.

\section{APPENDIX B: DETAILS OF NUMERICAL EXPERIMENTS}

The trajectory of the unknown target parameters (the $P$ $T-t$ path) was assumed to be monotonically increasing, from $520^{\circ} \mathrm{C}$ and $0.8 \mathrm{GPa}$ at $0 \mathrm{Myr}$ to $600^{\circ} \mathrm{C}$ and $1.05 \mathrm{GPa}$ at 2.0 Myr, and to be convex downward, as shown in Figs. 4(a)4(c). The synthetic garnet grain grew according to the assumed diffusion-controlled grain-growth law [Eq. (18)] along the assumed $P-T-t$ trajectory. The parameter values were taken from Okudaira's previous work [28]: the rate constant $k$ was $6.29 \times 10^{-14} \mathrm{~m}^{2} / \mathrm{s}$; the activation energy $E_{a}$ was
$8.37 \times 10^{4} \mathrm{~J} / \mathrm{mol}$; and the gas constant $R$ was $8.314 \mathrm{~J} /(\mathrm{mol} \mathrm{K})$. Figure 4(d) shows the composition of a synthetic garnet grain.

The thermodynamic system was assumed to be according to Kuwatani et al. [26]. There, a mineral combination consisting of garnet, biotite, chlorite, plagioclase, epidote, muscovite, quartz, and water was considered to form the chemical system $\mathrm{SiO}_{2}-\mathrm{Al}_{2} \mathrm{O}_{3}-\mathrm{MgO}-\mathrm{FeO}-\mathrm{CaO}-\mathrm{Na}_{2} \mathrm{O}-\mathrm{K}_{2} \mathrm{O}-\mathrm{H}_{2} \mathrm{O}$. Since there are eight system components and eight phases, the Gibbs phase rule variance is 2 . This determined the chemical composition of the mineral for a given temperature $T$ and pressure $P$, i.e., it was fully determined by the external conditions, pressure, and temperature. For this system, the thermodynamic functions are $X_{\mathrm{Fe}}=\xi_{\mathrm{Fe}}(T, P)$ and $X_{\mathrm{Ca}}=\xi_{\mathrm{Ca}}(T, P)$.

The prior PDF, which determines the initial conditions of the numerical simulation, was assumed to be

$$
p\left(z_{0}\right) \sim N\left[\left(T_{0}, P_{0}, r_{0}^{2}\right)^{\top}, \operatorname{diag}\left(\sigma_{0, T}^{2}, \sigma_{0, P}^{2}, 0\right)^{\top}\right],
$$

where $r_{0}, T_{0}$, and $P_{0}$ were assumed to be $0 \mathrm{~mm}, 520^{\circ} \mathrm{C}$, and $0.75 \mathrm{GPa}$, and the standard deviations $\sigma_{0, T}$ and $\sigma_{0, P}$ were assumed to be $50^{\circ} \mathrm{C}$ and $0.05 \mathrm{GPa}$. We assumed that the standard deviations of the random walks for temperature $\left(\sigma_{\Delta T}\right)$ and pressure $\left(\sigma_{\Delta P}\right)$ were $20^{\circ} \mathrm{C}$ and $0.01 \mathrm{GPa}$, respectively. The standard deviation of the system noise for the growth law $\sigma_{\Delta r^{2}}$ was assumed to be $(0.1 \mathrm{~mm})^{2}$ per step, corresponding to $0.01 \mathrm{Myr}$.
[1] E. Kalnay, Atmospheric Modeling, Data Assimilation, and Predictability (Cambridge University Press, Cambridge, 2003).

[2] G. Evensen, Data Assimilation: The Ensemble Kalman Filter (Springer Science \& Business Media, Berlin, 2009).

[3] M. Hoshiba and S. Aoki, Numerical shake prediction for earthquake early warning: Data assimilation, real-time shake mapping, and simulation of wave propagation, Bull. Seismol. Soc. Am. 105, 1324 (2015).

[4] R. Niwayama, H. Nagao, T. S. Kitajima, L. Hufnagel, K. Shinohara, T. Higuchi, T. Ishikawa, and A. Kimura, Bayesian inference of forces causing cytoplasmic streaming in Caenorhabditis elegans embryos and mouse oocytes, PLoS ONE 11, e0159917 (2016).

[5] T. Omori, T. Kuwatani, A. Okamoto, and K. Hukushima, Bayesian inversion analysis of nonlinear dynamics in surface heterogeneous reactions, Phys. Rev. E 94, 033305 (2016).

[6] S. Ito, H. Nagao, A. Yamanaka, Y. Tsukada, T. Koyama, M. Kano, and J. Inoue, Data assimilation for massive autonomous systems based on a second-order adjoint method, Phys. Rev. E 94, 043307 (2016).

[7] I. K. Shimatani and Y. Kubota, The spatio-temporal forest patch dynamics inferred from the fine-scale synchronicity in growth chronology, J. Veg. Sci. 22, 334 (2011).

[8] S. Nakano, K. Suzuki, K. Kawamura, F. Parrenin, and T. Higuchi, A sequential Bayesian approach for the estimation of the age-depth relationship of the Dome Fuji ice core, Nonlin. Process. Geophys. 23, 31 (2016).

[9] C. E. Rasmussen and C. K. Williams, Gaussian Processes for Machine Learning (MIT Press, Cambridge, 2005), Vol. 1.
[10] F. S. Spear, Metamorphic Phase Equilibria and PressureTemperature-time Paths (Mineralogical Society of America, Washington, 1993).

[11] A. Spry, in Metamorphic Textures (Pergamon Press, New York, 1969), p. 350.

[12] R. H. Vernon, A Practical Guide to Rock Microstructure (Cambridge University Press, Cambridge, 2004).

[13] H. Rollinson, Metamorphic history suggested by garnet-growth chronologies in the Isua Greenstone Belt, West Greenland, Precambr. Res. 126, 181 (2003).

[14] T. Hassenkam, M. Andersson, K. Dalby, D. Mackenzie, and M. Rosing, Elements of Eoarchean life trapped in mineral inclusions, Nature 548, 78 (2017).

[15] A. C. Lasaga, Kinetic Theory in the Earth Sciences (Princeton University Press, Princeton, NJ, 1998).

[16] B. W. Yardley, An empirical study of diffusion in garnet, Am. Mineral. 62, 793 (1977).

[17] G. Kitagawa, Introduction to Time Series Modeling, CRC Monographs on Statistics \& Applied Probability (Chapman \& Hall/CRC Press, New York, 2010).

[18] P. S. Swain, K. Stevenson, A. Leary, L. F. Montano-Gutierrez, I. B. Clark, J. Vogel, and T. Pilizota, Inferring time derivatives including cell growth rates using Gaussian processes, Nat. Commun. 7, 13766 (2016).

[19] Y. Igarashi, T. Hori, S. Murata, K. Sato, T. Baba, and M. Okada, Maximum tsunami height prediction using pressure gauge data by a Gaussian process at Owase in the Kii Peninsula, Japan, Marine Geophys. Res. 37, 361 (2016).

[20] M. R. St-Onge, Zoned poikiloblastic garnets: PT paths and synmetamorphic uplift through $30 \mathrm{~km}$ of structural depth, Wopmay Orogen, Canada, J. Petrol. 28, 1 (1987). 
[21] D. Rubatto, D. Regis, J. Hermann, M. Engi, M. Beltrando, and S. R. McAlpine, Yo-yo subduction recorded by accessory minerals in the Italian Western Alps, Nat. Geosci. 4, 338 (2011).

[22] J. Blundy, K. Cashman, and M. Humphreys, Magma heating by decompression-driven crystallization beneath andesite volcanoes, Nature 443, 76 (2006).

[23] C. Ginibre, G. Wörner, and A. Kronz, Crystal zoning as an archive for magma evolution, Elements 3, 261 (2007).

[24] K. Saunders, J. Blundy, R. Dohmen, and K. Cashman, Linking petrology and seismology at an active volcano, Science 336, 1023 (2012).

[25] D. A. Budd, V. R. Troll, F. M. Deegan, E. M. Jolis, V. C. Smith, M. J. Whitehouse, C. Harris, C. Freda, D. R. Hilton, S. A. Halldórsson, and I. N. Bindeman, Magma reservoir dynamics at Toba caldera, Indonesia, recorded by oxygen isotope zoning in quartz, Sci. Rep. 7, 40624 (2017).

[26] T. Kuwatani, K. Nagata, M. Okada, and M. Toriumi, Precise estimation of pressure-temperature paths from zoned minerals using Markov random field modeling: Theory and synthetic inversion, Contrib. Mineral. Petrol. 163, 547 (2012).

[27] D. Vance and R. O'nions, Isotopic chronometry of zoned garnets: Growth kinetics and metamorphic histories, Earth Planet. Sci. Lett. 97, 227 (1990).

[28] T. Okudaira, Temperature-time path for the low-pressure Ryoke metamorphism, Japan, based on chemical zoning in garnet, J. Metamorph. Geol. 14, 427 (1996).
[29] M. Tirone and J. Ganguly, Garnet compositions as recorders of P-T-t history of metamorphic rocks, Gondwana Res. 18, 138 (2010).

[30] K. V. Hodges and L. McKenna, Realistic propagation of uncertainties in geologic thermobarometry, Am. Mineral. 72, 671 (1987).

[31] M. J. Kohn, Uncertainties in differential thermodynamic (Gibbs' method) PT paths, Contrib. Mineral. Petrol. 113, 24 (1993).

[32] G. Kitagawa, Non-Gaussian state-space modeling of nonstationary time series, J. Am. Stat. Assoc. 82, 1032 (1987).

[33] See Supplemental Material at http://link.aps.org/supplemental/ 10.1103/PhysRevE.98.043311 for estimated results and video file.

[34] M. J. Kohn and F. S. Spear, Error propagation for barometers: 2. Application to rocks, Am. Mineral. 76, 138 (1991).

[35] C. M. Bishop, Pattern Recognition and Machine Learning (Springer-Verlag, Berlin, 2006).

[36] P. Bisaillon, R. Sandhu, M. Khalil, C. Pettit, D. Poirel, and A. Sarkar, Bayesian parameter estimation and model selection for strongly nonlinear dynamical systems, Nonlin. Dyn. 82, 1061 (2015).

[37] N. Van Kampen, Stochastic Processes in Physics and Chemistry (North Holland, Amsterdam, 2007).

[38] Y. Igarashi, K. Nagata, T. Kuwatani, T. Omori, Y. NakanishiOhno, and M. Okada, Three levels of data-driven science, J. Phys.: Conf. Ser. 699, 012001 (2016). 